\title{
A facile one pot synthesis of bisphosphonic acids and their sodium salts from nitriles
}

\author{
Divvela V. N. Srinivasa Rao, ${ }^{a}$ Ramesh Dandala, ${ }^{a *}$ Racha Lenin, \\ Meenakshisunderam Sivakumaran, ${ }^{a}$ Sripelly Shivashankar, ${ }^{a}$ and Andra Naidu ${ }^{b}$ \\ ${ }^{a}$ Chemical Research Department, APL Research Centre, Hyderabad-500 072, India \\ ${ }^{b}$ J. N. T. University, Kukatpally, Hyderabad-500 072, Andhra pradesh, India \\ E-mail: rdandala@aurobindo.com
}

\begin{abstract}
A general and one pot synthesis for the preparation of bisphosphonic acids and their sodium salts (2a-e) from nitriles (3a-e) is described. This method involves hydrolysis of nitriles (3a-e) to the corresponding acids and subsequent bisphosphonation in a single solvent to produce bisphonates (2a-e). Preparations of some of bisphosphonates, which are presently in clinical use like risedronate (2a) sodium, ibandronate sodium (2d) are synthesized by following this new method. This method is useful for the preparation of other bisphosphonate compounds.
\end{abstract}

Keywords: Bisphosphonates, pamidronate, risedronate, ibandronate, alendronate, zolendronate

\section{Introduction}

Bisphosphonic acids and/or their salts are excellent antihypercalcemics and as such are rapidly evolving as therapeutic agents for the treatment of a number of diseases which are characterized by abnormal calcium metabolism. ${ }^{1}$ Bisphosphonates, in particular bisphosphonates, 1-hydroxy2-(pyridinyl)ethylidene-1,1-bisphosphonic acid (risedronic acid 2a), 1-hydroxy-3-(methylpentylamino)propylidene bisphosphonic acid (ibandronate 2d), 3-amino-1-hydroxy propylidene bisphosphonic acid (pamidronate), 4-amino-1-hydroxybutylidene-1,1-bisphosphonic acid (alendronate) are used for the treatment of Paget's disease of bone and osteoporosis. The synthesis of 1-hydroxyalkylidene-1,1-bisphosphonic acids (bisphosphonates) is based on reacting a carboxylic acid (1) with a mixture of phosphorous acid and phosphorous chloride such as $\mathrm{PCl}_{3}$ or $\mathrm{PCl}_{5}$, then quenching the reaction mixture with water followed by heating to hydrolyze the phosphonated reaction mass to get required product (2). 1-Hydroxyalkylidene-1,1 bisphosphonic acids were prepared using a solvent such as chlorobenzene, ${ }^{2}$ methanesulphonic acid, ${ }^{3}$ sulfolane, ${ }^{4}$ ionic liquids ${ }^{5}$ and diphenyl ether ${ }^{6}$ as shown in Scheme 1. Our present work 
envisages a new and direct method for the synthesis of bisphosphonic acids and their salts (2a-e) from nitrile compounds (3a-e) as a one-pot synthesis.

\section{Results and Discussion}

In our efforts to develop a simple and industrial feasible method for the synthesis of risedronic acid 2a, experiments were carried out with known methods in the literature. ${ }^{2-6}$ In all these methods (Scheme 1), it was obtained by the reaction of 3-pyridylacetic acid with phosphorous acid and phosphorous trichloride in a solvent such as chlorobenzene, ${ }^{2}$ methanesulfonic acid, ${ }^{3}$ sulfolane, ${ }^{4}$ ionic liquids, ${ }^{5}$ and diphenyl ether. ${ }^{6}$ All the above processes have some disadvantages associated with their use such as solid mass formation which prevents uniform mixing. However methanesulphonic acid is helpful for solubilizing the reaction mass but it gave lower yield (40\%, reported $^{3} 38 \%$ ). Moreover they reported ${ }^{2-6}$ the yields $50 \%, 38 \%, 70 \%, 49 \%$ and $77 \%$ respectively.

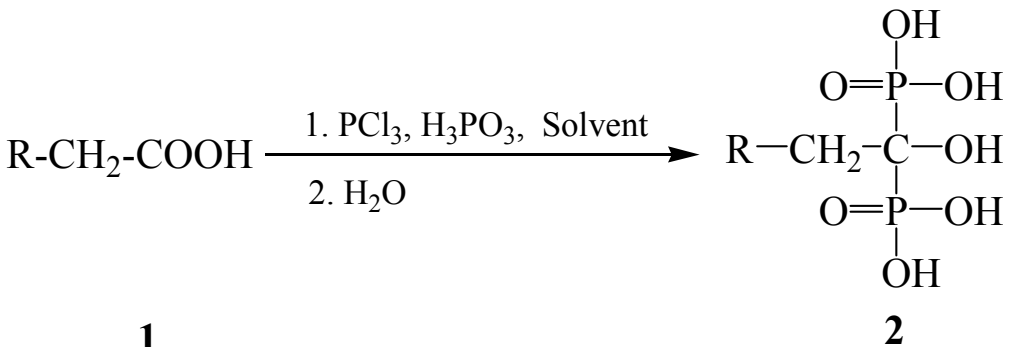

\section{Scheme 1}

Because of the therapeutic importance of this class of compounds, it became essential to develop an efficient and practical synthesis method. We report a new simple procedure to synthesize risedronic acid $\mathbf{2 a}$ with a better yield 79\% and its sodium salt $71 \%$ directly from 3 pyridinecarbonitrile 3a in a suitable solvent (Scheme 2). Hydrolysis of 3-pyridinecarbonitrile 3a in aqueous methanesulfonic acid $^{7}$ produces the corresponding carboxylic acid in situ and is allowed to react with phosphorous trichloride and the resulting phosphonated reaction mass on further hydrolysis yielded the bisphosphonic acids at $\mathrm{pH}<2$ or its monosodium salt at $\mathrm{pH}$ 4.24.5 in good yields. The commercially available aqueous methanesulfonic acid (85\%) is cheaper than methanesulfonic acid and is used both as acid catalyst in the first operation and as solvent in the subsequent phosphonylation reaction. This process is industrially much favored because the entire process is carried out in a single solvent which itself promotes the acid catalyzed hydrolysis of nitrile group. Further, to evaluate this methodology we have tried to prepare various bisphosphonates and got the required product in good yield. 


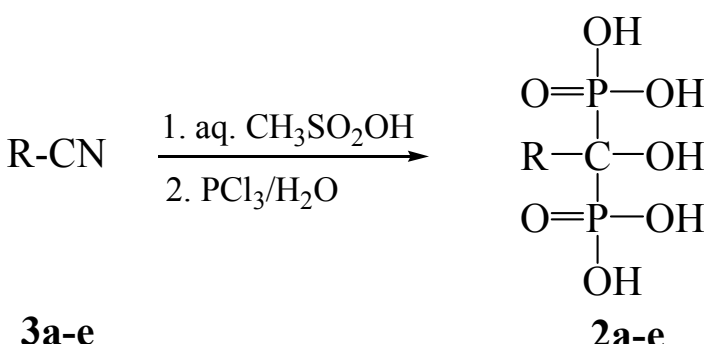

3a and $2 \mathbf{a} \mathrm{R}=\overbrace{\mathrm{N}}^{\mathrm{CH}_{2}}$

3c and $2 \mathrm{c} \mathrm{R}=$

3e and $2 \mathbf{e} R=$
$3 \mathbf{b}$ and $\mathbf{2 b} \mathrm{R}=\mathrm{N}_{\mathrm{N} \mathrm{H}_{2}}$

3d and $\mathbf{2 d} \mathrm{R}=\overbrace{}^{\mathrm{N}} \sim \mathrm{CH}_{2}$

\section{Scheme 2}

In summary, a one-pot synthesis of bisphosphonic acids and/or sodium salts (2a-e) from corresponding nitriles (3a-e) is described. The inexpensive and readily available starting material and reaction solvent make this procedure a practical and simple one-pot method for the preparation of bisphosphonic acid and/or its sodium salt. Finally, this methodology can be used for making other bisphosphonate drugs such as zoledronic acid and minodronic acid.

\section{Experimental Section}

General Procedures. The IR spectra were recorded on a Perkin Elmer Spectrum of FTIR spectrometer. ${ }^{1} \mathrm{H}-\mathrm{NMR}$ and ${ }^{13} \mathrm{C}-\mathrm{NMR}$ spectra were recorded on a Bruker $300 \mathrm{MHz}$ and $75 \mathrm{MHz}$ spectrometer respectively. Chemical shifts are reported in ppm downfield from TMS as internal standard. Mass spectra were measured on Perkin Elmer PE SCIEX-API 2000 mass spectrometer.

1-Hydroxy-2-(3-pyridyl)ethylidene bisphosphonic acid (risedronic acid, 2a). Aqueous methanesulfonic acid $(85 \%, 432.5 \mathrm{~mL})$ was added to 3 -pyridinecarbonitrile ${ }^{8}$ 3a $(100.0 \mathrm{~g}, 0.847$ mol) and heated to $98-100{ }^{\circ} \mathrm{C}$ for $8 \mathrm{~h}$. Then, cooled the reaction temperature to $65{ }^{\circ} \mathrm{C}$ and phosphorus trichloride $(396.0 \mathrm{~g}, 2.880 \mathrm{~mol})$ was added over $25 \mathrm{~min}$. After $5 \mathrm{~h}$. stirring at 65-70 ${ }^{\circ} \mathrm{C}$, the reaction mass temperature was cooled to $30{ }^{\circ} \mathrm{C}$ and pre-cooled water $(1000 \mathrm{~mL})$ was added very slowly in $30 \mathrm{~min}$. The reaction mass temperature was heated to $98{ }^{\circ} \mathrm{C}$. After $15 \mathrm{~h}$ stirring, the temperature was cooled to $50{ }^{\circ} \mathrm{C}$ and methanol $(1500 \mathrm{~mL})$ was added. After $2 \mathrm{~h}$. stirring at $5-10{ }^{\circ} \mathrm{C}$, the product was collected by filtration and dried to yield $\mathbf{2 a}(201.5 \mathrm{~g}, 79 \%)$ as 
a white monohydrate solid; purity ${ }^{9} 99.1 \%$ (by HPLC); IR $\left(\mathrm{KBr}, \mathrm{cm}^{-1}\right) 3189,3091,3069,1635$, 1617, 1262, 1073; ${ }^{1} \mathrm{H}$ NMR $\left(\mathrm{D}_{2} \mathrm{O} / \mathrm{NH}_{3}\right) \delta 3.26(\mathrm{t}, 2 \mathrm{H}, J=12.1 \mathrm{~Hz}), 7.54$, (dd, $\left.1 \mathrm{H}, J=8.2,5.5 \mathrm{~Hz}\right)$, $8.18(\mathrm{~d}, 1 \mathrm{H}, J=8.2 \mathrm{~Hz}), 8.32(\mathrm{~d}, 1 \mathrm{H}, J=5.5 \mathrm{~Hz}), 8.51,(\mathrm{~s}, 1 \mathrm{H}) ;{ }^{13} \mathrm{C}-\mathrm{NMR}\left(300 \mathrm{MHz}, \mathrm{D}_{2} \mathrm{O} / \mathrm{NH}_{3}\right) \delta$ $36.5,73.8,125.3,137.1,141.3,145.4,146.1 ;{ }^{31} \mathrm{P}$ NMR $\left(\mathrm{D}_{2} \mathrm{O} / \mathrm{NH}_{3}\right) \delta 18.1 ; \mathrm{MS}(\mathrm{ESI}, m / z): 282.0$ $[\mathrm{M}-\mathrm{H}]^{+}$.

Risedronic acid monosodium salt (2a sodium). Monosodium salt was obtained by the above procedure with 3-pyridinecarbonitrile 3a (100.0 g, $0.847 \mathrm{~mol})$. But, $\mathrm{pH}$ was adjusted to 4.3 with $30 \%$ sodium hydroxide solution before diluting with methanol $(1500 \mathrm{~mL})$. The resulting product was filtered and dried to yield 2 a sodium $(210 \mathrm{~g}, 71 \%)$ as a white crystalline hemipentahydrate solid; purity ${ }^{9} 99.8 \%$ (by HPLC); IR $\left(\mathrm{KBr}, \mathrm{cm}^{-1}\right)$ 3621, 3566, 3364, 1689, 1655, 1211, 1134, 1065; ${ }^{1} \mathrm{H}$ NMR $\left(\mathrm{D}_{2} \mathrm{O}\right) \delta 3.37$ (t, 2H, J=12.1 Hz), 7.84, (m, 1H), 8.50 (d, 2H, J=7.2 Hz), 8.66 (s, $1 \mathrm{H}) ;{ }^{13} \mathrm{C}-\mathrm{NMR}\left(300 \mathrm{MHz}, \mathrm{D}_{2} \mathrm{O}\right) \delta 36.4,73.9,126.3,138.4,138.9,142.7,149.4 ;{ }^{31} \mathrm{P}$ NMR $\left(\mathrm{D}_{2} \mathrm{O}\right)$ $\delta 17.1$; MS (ESI, $m / z): 284.1[\mathrm{M}+\mathrm{H}]^{+}$.

1-Hydroxy-2-(2-pyridyl)ethylidene bisphosphonic acid monosodium (2b). This compound was prepared in a similar way to 2a sodium, using 2-pyridinecarbonitrile $\mathbf{3 b}(10.0 \mathrm{~g}, 0.085 \mathrm{~mol})$ procured from Aldrich chemicals, as a white hemipentahydrate solid (21 g, 71\%); purity ${ }^{9} 99.4 \%$ (by HPLC); IR (KBr, cm ${ }^{-1}$ ) 3190, 3186, 1638, 1625, 1215, 1058; ${ }^{1} \mathrm{H}$ NMR (D $\left.2 \mathrm{O}\right) 3.54$ (t, 2H, $J=12.9 \mathrm{~Hz}$ ), 7.73 (dd, 1H, $J=7.9,7.1 \mathrm{~Hz}), 7.82$, (d, 1H, $J=8.2 \mathrm{~Hz}), 8.29$, (dd, $1 \mathrm{H}, J=9.1,8.2 \mathrm{~Hz}$ ), $8.49(\mathrm{~d}, 1 \mathrm{H}, J=6.1 \mathrm{~Hz}) ;{ }^{13} \mathrm{C}-\mathrm{NMR}\left(300 \mathrm{MHz}, \mathrm{D}_{2} \mathrm{O}\right) \delta 37.7,73.3,125.1,129.8,140.7,145.9$, 153.4; ${ }^{31} \mathrm{P}$ NMR $\left(\mathrm{D}_{2} \mathrm{O}\right) \delta$ 17.0; MS (ESI, $\left.m / z\right): 284.1[\mathrm{M}+\mathrm{H}]^{+}$.

1-Hydroxy-2-(4-pyridyl)ethylidene bisphosphonic acid monosodium (2c). This compound was prepared in a similar way to 2 a sodium, using 4-pyridinecarbonitrile 3c (10.0 g, $0.085 \mathrm{~mol})$ procured from Aldrich chemicals, as a white monohydrate solid (20.5 g, 75\%); purity ${ }^{9}$ 99.2\% (by HPLC); IR (KBr, cm ${ }^{-1}$ ) 3242, 3107, 1644, 1626, 1224, 1058; ${ }^{1} \mathrm{H}$ NMR (D $\left.2 \mathrm{O}\right) 3.37$ (t, 2H, $J=12.1$ $\mathrm{Hz}), 7.88(\mathrm{~d}, 2 \mathrm{H}, J=6.0 \mathrm{~Hz}), 8.42(\mathrm{~d}, 2 \mathrm{H}, J=6.0 \mathrm{~Hz}) ;{ }^{13} \mathrm{C}-\mathrm{NMR}\left(300 \mathrm{MHz}, \mathrm{D}_{2} \mathrm{O}\right) \delta 39.6,74.5$, 129.6, 140.7, 159.2; ${ }^{31} \mathrm{P}$ NMR $\left(\mathrm{D}_{2} \mathrm{O}\right) \delta$ 17.2. MS (ESI, $\left.m / z\right): 284.1[\mathrm{M}+\mathrm{H}]^{+}$.

1-Hydroxy-3-(methylpentylamino)propylidene bisphosphonic acid monosodium (ibandronate sodium, 2d). This compound was prepared in a similar way to 2a sodium, but isolated from ethanol, using N-methylpentylamino propionitrile ${ }^{10} \mathbf{3 d}(50.0 \mathrm{~g}, 0.325 \mathrm{~mol})$ as a white monohydrate solid (75.4 g, 65\%); purity ${ }^{11} 99.2 \%$ (by HPLC); IR (KBr, cm ${ }^{-1}$ ) 3164, 2922, 2852, 1667, 1191, 1067; ${ }^{1} \mathrm{H}-\mathrm{NMR}\left(300 \mathrm{MHz}, \mathrm{D}_{2} \mathrm{O}\right) \delta 0.78$ (t, 3H, $\left.J=6.9 \mathrm{~Hz}\right), 1.21-1.25$ (m, 4H), $1.61-$ $1.63(\mathrm{~m}, 2 \mathrm{H}), 2.22-2.28(\mathrm{~m}, 2 \mathrm{H}), 2.74(\mathrm{~s}, 3 \mathrm{H}), 2.94-2.98(\mathrm{~m}, 1 \mathrm{H}), 3.10-3.25(\mathrm{~m}, 2 \mathrm{H}), 3.43-3.49$ $(\mathrm{m}, 1 \mathrm{H}) ;{ }^{13} \mathrm{C}-\mathrm{NMR}\left(300 \mathrm{MHz}, \mathrm{D}_{2} \mathrm{O}\right) \delta 13.4,21.8,23.5,28.1,28.2,39.7,53.2,56.7,72.6 ;{ }^{31} \mathrm{P}$ NMR $\left(\mathrm{D}_{2} \mathrm{O}\right) \delta$ 17.7; MS (ESI, $\left.m / z\right): 318.1[\mathrm{M}-\mathrm{H}]^{+}$.

1-Hydroxy-3-(pentylamino)propylidene bisphosphonic acid mono sodium (2e). This compound was prepared in a similar way to 2 a sodium, but isolated from ethanol, using Npentylamino propionitrile ${ }^{10} 3 \mathrm{e}(10.0 \mathrm{~g}, 0.071 \mathrm{~mol})$ as a white monohydrate solid $(15.7 \mathrm{~g}, 64 \%)$; purity $^{11} 99.1 \%$ (by HPLC); IR (KBr, cm ${ }^{-1}$ ) 3127, 2957, 2871, 1640, 1607, 1156, 1081; ${ }^{1} \mathrm{H}-\mathrm{NMR}$ $\left(300 \mathrm{MHz}, \mathrm{D}_{2} \mathrm{O}\right) \delta 0.79(\mathrm{t}, 3 \mathrm{H}, J=6.9 \mathrm{~Hz}), 1.23-1.25(\mathrm{~m}, 4 \mathrm{H}), 1.54-1.59(\mathrm{~m}, 2 \mathrm{H}), 2.14-2.27(\mathrm{~m}$, 
2H), 2.91-2.95 (m, 2H), 3.24-3.25 (m, 2H); ${ }^{13} \mathrm{C}-\mathrm{NMR}\left(300 \mathrm{MHz}, \mathrm{D}_{2} \mathrm{O}\right) \delta 13.4,21.8,25.7,28.2$, 30.0, 44.5, 48.1, 72.8; ${ }^{31} \mathrm{P}$ NMR $\left(\mathrm{D}_{2} \mathrm{O}\right) \delta 18.2$; MS (ESI, $\left.m / z\right): 306.2[\mathrm{M}+\mathrm{H}]^{+}$.

\section{Acknowledgements}

Authors thank the management of Aurobindo Pharma Limited, Hyderabad for permission to publish this work. Authors also thank the Analytical Research Department for their valuable contribution to this work.

\section{References and Footnotes}

1. (a) Geusens, P.; McClung, M. Expert Opin. Pharmacother. 2002, 2, 2011. (b) Dunn, C. J.; Goa, K. L. Drugs 2001, 61, 685.

2. Wiezorek, M.; Stawinski, T.; Chrulski, K.; Eur. Patent 1,243,592, 2005; Chem. Abstr. 2002, 137, 247819y.

3. Kieczykowski, G. R.; Jobson, R. B.; Melillo, D. G.; Reinhold, D. F.; Grenda, V. J.; Shinkai, I. J. Org. Chem. 1995, 60, 8310.

4. Vijay Kumar, P. M.; Trinadha Rao, Ch.; Rajamannar, T. PCT Int. Appl. WO 44,831, 2005; Chem. Abstr. 2005, 142, 463876k.

5. De Ferra, L.; Turchetta, S.; Massardo, P.; Casellato, P. PCT Int. Appl. WO 93,282, 2003; Chem. Abstr. 2003, 139, 365070m.

6. Despande, P. B.; Luthra, P. K. US Patent Appl. US 258,625, 2006; CAPLUS. 2006, 1204267.

7. Guangyu, X.; Yeyuam, X.; Xihan, W. Org. Prep. Proced. Int. 2004, 36, 185.

8. Mosher, H. S.; Tessieri, J. E. J. Am. Chem. Soc. 1951, 73, 4925.

9. Analytical HPLC were run with Zorbax SB-C8, $5 \mu(150$ X $4.6 \mathrm{~mm})$ column for compounds $2 \mathrm{a}, 2 \mathrm{~b}$, and $2 \mathrm{c}$ at $262 \mathrm{~nm}$. Retention times were $8.9,4.5$ and 8.1 respectively $\left(\mathrm{CH}_{3} \mathrm{CN}: 0.15\right.$ $\left.\mathrm{M} \mathrm{Na}_{2} \mathrm{HPO}_{4}: 0.01 \mathrm{M}\left[\left(\mathrm{C}_{4} \mathrm{H}_{9}\right) \mathrm{N}^{+} \mathrm{Br}^{-}\right)\right]$.

10. Szabo, C. M.; Matsumura, M.; Fukura, S.; Martin, M. V.; Sanders, J. M.; Sengupta, S.; Cieslak, J. A.; Loftus, T. C.; Lea, C. R.; Lee, H.-J.; Koohang, A.; Coates, R. M.; Sangami, Hiroshi.; Oldfield, E. J. Med. Chem. 2002, 45, 2185.

11. Analytical HPLC were run with Intersil C $8-3,5 \mu(250$ X $4.6 \mathrm{~mm})$ column for compounds $2 \mathrm{~d}$ and 2e using Refractive Index Detector. Retention times were 6.7 and 6.6 respectively $\left(\mathrm{CH}_{3} \mathrm{OH}: 0.2 \mathrm{M} \mathrm{CH}_{3} \mathrm{COONH}_{4}\right)$. 